\title{
ON ORDERED POLYCYCLIC GROUPS
}

\author{
R. J. HURSEY, JR.
}

ABstract. It has been asserted that any (full) order on a torsion-free, finitely generated, nilpotent group is defined by some $F$-basis of $G$ and that the group of $o$-automorphisms of such a group is itself a group of the same kind: Examples provided herein demonstrate that both of these assertions are false; however, it is proved that the group of $o$-automorphisms of an ordered, polycyclic group is nilpotent by abelian, and polycyclic.

1. Introduction. It is well known that if $G$ is a torsion-free, finitely generated, nilpotent group, then $G$ possesses a central series $\{1\}$ $=F_{0} \subseteq F_{1} \subseteq \cdots \subseteq F_{m}=G$ such that $F_{i+1} / F_{i}$ is an infinite cyclic group, $i=0,1, \cdots, m-1$. Ree (see [5] and [6]) calls such a series an " $F$-series of $G$." It is also clear that if $F_{i+1} / F_{i}=\left\langle x_{i+1} F_{i}\right\rangle$, where $x_{i+1} \in F_{i+1}$ for $i=0,1, \cdots, m-1$, then each element $g$ of $G$ can be written uniquely in the form $g=x_{1}^{e_{1}} x_{2}^{e_{2}} \cdots x_{m}^{e_{m}}$, where $e_{1}, e_{2}, \cdots, e_{m}$ are integers. Ree [6] calls the elements $x_{1}, x_{2}, \cdots, x_{m}$ an " $F$-basis of $G$." It follows easily that the torsion-free, finitely generated, nilpotent group $G$ can be ordered lexicographically with respect to $e_{1}, \cdots, e_{m}$ as follows: For $g_{1}=x_{1}^{e_{1}} x_{2}^{e_{2}} \cdots x_{m}^{e_{m}}, g_{2}=x_{1}^{f_{1}} x_{2}^{f_{2}} \cdots x_{m}^{f_{m}}$, we put $g_{1} \leqq g_{2}$ if and only if $e_{i}=f_{i}$ for $i=1,2, \cdots, m$, or $e_{t}<f_{t}$ for some $t$ such that $1 \leqq t \leqq m$ and $e_{i}=f_{i}$ for $i=1,2, \cdots, t-1$. This lexicographic order on $G$ is said, by Ree [5], to be "defined" by the $F$-basis $x_{1}, x_{2}, \cdots, x_{m}$.

These concepts are used by Ree in [6], where it is asserted that any (full) order on a torsion-free, finitely generated, nilpotent group is defined by some $F$-basis of $G$, and in the proof of Theorem 2 [6], which asserts that the group of $o$-automorphisms of a torsion-free, finitely generated, nilpotent group is itself a torsion-free, finitely generated, nilpotent group. Both of these assertions, as shown by Examples 1 and 2 of this note, are false. While it is not possible to establish a result as strong as the one suggested by Theorem 2 of [6], we can prove

THEOREM. If $G$ is an ordered, polycyclic group and if $\Delta$ denotes the group of o-automorphisms of $G$, then $\Delta$ is nilpotent by abelian, and, moreover, $\Delta$ is polycyclic.

Received by the editors September 15, 1970.

A MS 1969 subject classifications. Primary 0675, 2022; Secondary 2018, 2040.

Key words and phrases. Polycyclic group, nilpotent group, cyclic normal series, $F$-series, $F$-basis; length of a polycyclic group.

Copyright (C) 1971, American Mathematical Society 
2. Definitions and notations. If $G$ is a group on which there can be defined a (full) order relation $\leqq$ with the property that $a, b, x, y \in G$ and $a \leqq b$ imply $x a y \leqq x b y$, then $G$ is said to be an ordered group and $\leqq$ is said to be an order on $G$. Associated with an order $\leqq$ on $G$ is the positive cone $P(G)$ of $G, P(G)=\{x \mid x \in G$ and $1 \leqq x\}$. It follows that the subset $P(G)$ of the ordered group $G$ has the following properties:

(i) $P(G) \cap P^{-1}(G)=\{1\}$;

(ii) $P(G) P(G) \subseteq P(G)$;

(iii) $x^{-1} P(G) x \subseteq P(G)$; and

(iv) $P(G) \cup P^{-1}(G)=G$.

Conversely, if $G$ is a group which possesses a subset $P(G)$ with properties (i)-(iv), then $G$ is an ordered group with respect to the relation $\leqq$ given by

$$
a \leqq b \text { if and only if } a^{-1} b \in P(G) .
$$

A subgroup $C$ of a group $G$ ordered with respect to $\leqq$ is convex (with respect to $\leqq$ ) if $g \in G, c \in C$, and $1 \leqq g \leqq c$ imply $g \in C$.

If $D \subset C$ are convex subgroups of the ordered group $G$ with the property that no convex subgroup of $G$ lies strictly between $D$ and $C$, then $D \prec C$ is a jump in the chain of convex subgroups of $G$.

If $G$ and $H$ are ordered groups and $f$ is a mapping of $G$ into $H$, then $f$ is an o-homomorphism of $G$ into $H$ if $f$ is a group homomorphism of $G$ into $H$ and $f$ is order-preserving in the sense that $a, b \in G$ and $a \leqq_{1} b$ imply $f(a) \leqq_{2} f(b)$, where $\leqq_{1}$ and $\leqq_{2}$ denote the orders on $G$ and $H$, respectively. Furthermore, if $f$ is a one-to-one o-homomorphism of $G$ onto $H$ and if $f^{-1}$ is an $o$-homomorphism of $H$ onto $G$, then $f$ is an $o$-isomorphism of $G$ onto $H$. An $o$-isomorphism of an ordered group $G$ onto $G$ is an o-automorphism of $G$.

If $G$ is a group, then the series $\{1\}=A_{0} \subseteq A_{1} \subseteq \cdots \subseteq A_{n}=G$ is a cyclic normal (invariant) series of $G$ if $A_{i}$ is a normal subgroup of $A_{i+1}(G)$ and $A_{i+1} / A_{i}$ is cyclic, $i=0,1, \cdots, n-1$. A group $G$ is polycyclic if $G$ possesses a cyclic normal series. Finally, by the length of a polycyclic group $G$, we mean the number of infinite cyclic factors $A_{i+1} / A_{i}$ in any cyclic normal series of $G$. It is well known that the length of a polycyclic group is an invariant for that group.

\section{Proofs.}

EXAMPLE 1. Let $G$ be the subgroup of the additive group of reals which is generated by $\{1, \sqrt{ } 2\}$; i.e., $G=\langle 1\rangle+\langle\sqrt{ } 2\rangle$. By restricting the natural Archimedean order on the reals to $G, G$ is an Archimedean ordered, finitely generated, abelian group, whence $G$ possesses no proper, nontrivial, convex subgroups.

Let $\{1\}=F_{0} \subseteq F_{1} \subseteq \cdots \subseteq F_{m}=G$ be an $F$-series of $G$ with corre- 
sponding $F$-basis $f_{1}, f_{2}, \cdots, f_{m}$. Then, as $G$ is polycyclic of length two, $m=2$, so that $\{1\}=F_{0} \subset F_{1} \subset F_{2}=G, F_{1}=\left\langle f_{1}\right\rangle$ and $F_{2} / F_{1}=\left\langle f_{2} F_{1}\right\rangle$. Thus, each element $g \in G$ can be written uniquely in the form $e_{1} f_{1}+e_{2} f_{2}=g$, where $e_{1}, e_{2}$ are integers. Let $\leqq_{1}$ denote the order on $G$ defined by the $F$-basis $f_{1}, f_{2}$.

Now suppose that $g=e_{1} f_{1}+e_{2} f_{2}$ and $0 \leqq{ }_{1} e_{1} f_{1}+e_{2} f_{2} \leqq e_{2}^{\prime} f_{2}$, where $e_{1}, e_{2}$, and $e_{2}^{\prime}$ are integers. Then $0 \leqq_{1}-e_{1} f_{1}+\left(e_{2}^{\prime}-e_{2}\right) f_{2}$. Thus, as $0 \leqq e_{1}$ and $0 \leqq-e_{1}, e_{1}=0$ and, hence, $g=e_{2} f_{2} \in\left\langle f_{2}\right\rangle$. Therefore, $\left\langle f_{2}\right\rangle$ is a proper, nontrivial, convex subgroup of $G$ with respect to $\leqq_{1}$, whence the $F$-basis $f_{1}, f_{2}$ cannot define the given Archimedean order on $G$.

EXAmple 2. Let $G=\left\langle a_{1}\right\rangle+\left\langle a_{2}\right\rangle+\left\langle a_{3}\right\rangle$, where $\left\langle a_{1}\right\rangle$ is an infinite cyclic group and where $\left\langle a_{2}\right\rangle+\left\langle a_{3}\right\rangle$ is isomorphic to the subgroup $H=\langle 1\rangle+\langle(1 / 2)(1+\sqrt{ } 5)\rangle$ of the additive group of reals. Let $P(G)$ $=\left\{n a_{1} \mid n\right.$ is a nonnegative integer $\} \cup\left\{x \mid x \in G-\left\langle a_{1}\right\rangle, x=r a_{1}+s a_{2}+t a_{3}\right.$, and $s+(t / 2)(1+\sqrt{ } 5)>0\}$. It readily follows that $P(G)$ defines an order on $G$, say $\leqq$, where for $x, y \in G, x \leqq y$ if and only if $(-x+y)$ $\in P(G)$. It is easy to see that $0<a_{1}, 0<a_{2}$, and $0<a_{3}$; also, $P(G)$ is not an Archimedean order on $G$ as $a_{1} \ll a_{2}$ and $a_{1} \ll a_{3}$ (i.e., $n a_{1}<a_{2}$, $n a_{1}<a_{3}$ for all integers $n$ ).

We now define two $o$-automorphisms of $G$ :

(i) $d: a_{1} \rightarrow a_{1}, a_{2} \rightarrow a_{3}, a_{3} \rightarrow a_{2}+a_{3}$,

(ii) $v: a_{1} \rightarrow a_{1}, a_{2} \rightarrow a_{2}+m a_{1}, a_{3} \rightarrow a_{3}+n a_{1}$,

where $m, n$ are arbitrary nonzero integers.

It follows easily that $d$ and $v$ are automorphisms of $G$. We now show that $d$ is order-preserving: Let $x=r a_{1}+s a_{2}+t a_{3}$ and suppose $0<x$. If $s=t=0$, then $x^{d}=r a_{1}>0$. Suppose, therefore, that $0 \neq s$ or $0 \neq t$. Then, without loss of generality, we may assume $r=0$, so that $x$ $=s a_{2}+t a_{3}$. Then $0<x$ is equivalent to $0<s+(t / 2)(1+\sqrt{ } 5)$, which is equivalent to $(-2 s) /(1+\sqrt{ } 5)<t$. On the other hand, $0<\left(s a_{2}+t a_{3}\right)^{d}$ $=t a_{2}+(s+t) a_{3}$ is equivalent to $3 t+t \sqrt{ } 5>-s(1+\sqrt{ } 5)$, which is true if and only if $t>-s(1+\sqrt{ } 5) /(3+\sqrt{ } 5)=-2 s /(1+\sqrt{ } 5)$. Thus, $d$ is order-preserving. An analogous argument establishes that $v$ is also order-preserving, whence $d$ and $v$ are $o$-automorphisms of $G$.

Let $\Delta=\langle d, v\rangle$, so that $\Delta$ is a subgroup of the group of $o$-automorphisms of $G$. It follows easily that $v=[v, d]^{d}$, whence $v \neq 1$ belongs to each term of the lower central series of $\Delta$. Therefore, $\Delta$ is not nilpotent, and, as $\Delta$ is a subgroup of the group of $o$-automorphisms of $G$, the group of $o$-automorphisms of the torsion-free, finitely generated, abelian group $G$ is not nilpotent.

We proceed now with the

Proof of THE Theorem. Let $\{1\} \prec C_{1} \prec C_{2} \cdots \prec C_{n}=G$ be the chain of convex subgroups of $G$ with respect to the given order on $G$. 
We observe here that as $G$ satisfies the maximal condition for subgroups, this chain is necessarily of finite length and that this chain is an invariant series of $G$ (see [3]). Let $\theta \in \Delta$. Then $\theta$ induces an $o$-automorphism on the ordered group $C_{i} / C_{i-1}$, for $i=1,2, \cdots, n$, given by $\left(c C_{i-1}\right)^{\theta^{\prime}}=c^{\theta} C_{i-1}$.

For each $i$ such that $1 \leqq i \leqq n$, let $\Delta_{i}$ denote the group of all $o$-automorphisms $\theta \in \Delta$ such that $\theta^{\prime}$ centralizes $C_{i} / C_{i-1}$, i.e., such that $\left(c C_{i-1}\right)^{\theta^{\prime}}=c^{\theta} C_{i-1}=c C_{i-1}$. Then $\Delta_{i}$ is a normal subgroup of $\Delta$ for each $i$, and $\Delta / \Delta_{i}$ is isomorphic to a subgroup of the group of $o$-automorphisms of $C_{i} / C_{i-1}$. But, each $C_{i} / C_{i-1}$ is an Archimedean ordered group (see $[1, \mathrm{p} .50])$, whence the group of $o$-automorphisms of $C_{i} / C_{i-1}$ is isomorphic to a subgroup of the multiplicative group of positive real numbers (see [1, Corollary 3, p. 47]). Thus, $\Delta / \Delta_{i}$ is abelian for $i$ $=1,2, \cdots, n$. Hence, $\Delta / \Delta_{0}$ is abelian, where $\Delta_{0}=\bigcap_{i=1}^{n} \Delta_{i}$. Note that $\Delta_{0}$ centralizes $C_{i} / C_{i-1}$ for each $i$, so that $\left[C_{i}, \Delta_{0}\right] \subseteq C_{i-1}, i=1,2, \cdots, n$. Thus, by a result of P. Hall (see [2, Corollary to Theorem 3.8, p. 10]), $\Delta_{0}$ is nilpotent. Therefore, $\Delta$ is nilpotent by abelian.

Smirnov [7] has proved that, for a polycyclic group $H$, every abelian subgroup of $\operatorname{Aut}(H)$ is finitely generated, whereas Mal'cev [4] has proved that any solvable group, all of whose abelian subgroups are finitely generated, is polycyclic. These results prove that $\Delta$ is polycyclic.

As a final remark, I should like to thank Professors R. D. Bercov and A. H. Rhemtulla for their invaluable assistance in the preparation of this paper and for the many fruitful discussions while this author was a student at the University of Alberta.

\section{REFERENCES}

1. L. Fuchs, Partially ordered algebraic systems, Pergamon Press, New York; Addison-Wesley, Reading, Mass., 1963. MR 30 \#2090.

2. P. Hall, Nilpotent groups, Lecture Notes, Fourth Canadian Math. Congress, 1957.

3. R. J. Hursey, Jr., and A. H. Rhemtulla, Ordered groups satisfying the maximal condition locally, Canad. J. Math. 22 (1970), 753-758.

4. A. I. Mal'cev, On certain classes of infinite solvable groups, Mat. Sb. 28 (70) (1951), 567-588; English transl., Amer. Math. Soc. Transl. (2) 2 (1956), 1-21. MR 13, 203.

5. R. Ree, On ordered, finitely generated, solvable groups, Trans. Roy. Soc. Canad. Sect. III (3) 48 (1954), 39-42. MR 16, 792.

6. - The existence of outer automorphisms of some groups. II, Proc. Amer. Math. Soc. 9 (1958), 105-109. MR $20 \# 912$.

7. D. M. Smirnov, On a class of infinite solvable groups, Ivanov. Gos. Ped. Inst. Uč. Zap. Fiz.-Mat. Nauki 5 (1954), 57-60. (Russian) MR 17, 709.

East Carolina University, Greenville, North Carolina 27834 